\title{
DOCTRINA DE GALENO SOBRE LAS CAUSAS EN LOS PULSOS. IV: CASOS Y COMENTARIOS*
}

\author{
Luis Miguel Pino Campos \\ Universidad de La Laguna \\ $\underline{\text { lmpino@ull.edu.es }}$
}

In memoriam ISABEL GARCÍA GÁLVEZ ${ }^{1}$

\begin{abstract}
RESUMEN
Este es el cuarto estudio que dedicamos al tratado de Galeno titulado Sobre las causas en los pulsos, en el que analizamos las causas "alteradoras" del pulso cuando es una sola anomalía la que afecta a la facultad (corazón) o a los órganos (arterias).

Palabras Clave: Medicina, Galeno, Esfigmología, Causas en los pulsos anómalos.
\end{abstract}

\section{ABSTRACT}

"The doctrine of Galen about the causes in the pulses. IV: cases and comments». In my fourth paper devoted to the Galen's treatise entitled About the causes in the pulses, the "disturbing" causes of the pulse when there is only an anomaly affecting the faculty (heart) or the organs (arteries) have been analyzed.

KEY WORDS: Medicine, Galen, Sphygmology, Causes in anomalous pulses.

\section{INTRODUCCIÓN}

En tres estudios anteriores hemos analizado y comentado el contenido

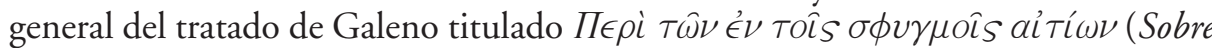
las causas en los pulsos). En el primer estudio ofrecimos una introducción al contenido del tratado. En el segundo hemos hecho una síntesis del libro primero, en el que Galeno explica los tipos de causas que cabe distinguir en los pulsos, cuales son el tipo de causas generadoras y el tipo de causas alteradoras; dicho libro primero se ocupa de explicar las causas primeras, denominadas "generadoras", porque generan o producen pulsos diferentes por alguna afección de la facultad (localizada en el corazón) o de los órganos (entendidos como las arterias). En el tercer estudio hemos descrito y comentado una parte del contenido del libro segundo, en concreto, el contenido de los capítulos I-IV, en el que Galeno explica las causas alteradoras del pulso que se producen por varias anomalías simultáneas, las cuales afectan tanto a la facultad (corazón) como a los órganos (arterias). En una segunda parte que desarrollamos en este estudio y que comprende el contenido del libro segundo desde el capítulo quinto hasta el 
final, Galeno se ocupa de describir las causas alteradoras de los pulsos producidas por una única anomalía.

\section{LOS PULSOS ANÓMALOS POR UNA SOLA ALTERACIÓN: LA PAUSA DIASTÓLICA}

En los capítulos quinto y sexto del libro segundo del tratado que comentamos, Galeno empieza a describir los pulsos que presentan una sola anomalía en la facultad (corazón) o en los órganos (arterias). Por ejemplo, dentro de los cuatro tiempos en los que cabe dividir un latidoº el primero es la diástole; a esta seguirán la pausa postdiastólica, la sístole y la pausa postsistólica.

La diástole normal y sana es aquella que se produce sin interrupción: se dilata todo lo que debe y finaliza con el comienzo de la pausa postdiastólica; el movimiento pulsístico continuará con la contracción arterial (o sístole) y finalizará con la pausa postsistólica; a continuación, se inicia un nuevo latido con sus cuatro tiempos, y así será mientras el pulso funcione con normalidad en un cuerpo que esté sano.

Ahora bien, cuando hay alguna anomalía, el movimiento diastólico que debería ser continuo, puede padecer una interrupción en unos instantes de la dilatación arterial (entiéndase unas décimas de segundo) y pocos instantes después vuelve a reanudar la dilatación hasta completarla; tras ella seguirán la pausa postdiastólica, la sístole y la pausa postsistólica.

Pues bien, fijando la atención en una diástole interrumpida por una pausa, cabe distinguir en ella tres partes: a) comienzo de la diástole, b) interrupción de la dilatación arterial y c) reanudación del movimiento de dilatación arterial hasta su final; tras este, continuará la primera pausa (pausa postdiastólica) y le seguirán la sístole o contracción arterial y la pausa postsistólica.

Para captar bien esta alteración del pulso se ha de fijar la atención en el tiempo de la diástole, que es el tiempo del movimiento arterial que mejor se percibe en el tacto. Si este tiempo diastólico se viera interrumpido por una parada en medio de la dilatación, y luego se reanudara esa dilatación arterial, tendríamos una alteración del pulso considerada en una anomalía única.

* Este estudio forma parte del Proyecto de Investigación FFI2014-55220-R, 2015-2017, financiado por el Ministerio de Economía y Competitividad.

${ }^{1}$ En los últimos años nuestro Departamento ha perdido a varios compañeros mientras estaban aún en activo: Inmaculada, Eduardo e Isabel. Aunque han pasado varios años, su recuerdo permanece vivo en nuestra memoria. Sea este modesto estudio una muestra de nuestra amistad, truncada por el destino, con ocasión del merecido homenaje que la revista Fortunatae dedica en número extraordinario a Isabel García Gálvez, quien se esforzó desde su llegada a la Universidad de La Laguna por difundir la enseñanza del griego clásico y del griego moderno. La Universidad de La Laguna estaba en deuda con ella y hoy se une junto con colegas de otras universidades españolas y extranjeras a este merecido recuerdo que en forma de libro le dedicamos.

${ }^{2}$ Primer tiempo: diástole o dilatación arterial; segundo tiempo: pausa postdiastólica; tercer tiempo: sístole o contracción arterial, y cuarto tiempo: pausa postsistólica. 
La combinación de esos tres momentos de la diástole (inicio de la diástole, parada de la diástole y reanudación de la diástole hasta el inicio de la pausa postdiastólica) son los indicios de una anomalía. Galeno añade que analizando esos tres momentos se puede concluir si el primer movimiento, antes de que se pare la diástole en medio de la dilatación, es rápido, moderado o lento; y también se puede establecer si el segundo movimiento de dilatación que sigue a esa parada diastólica es rápido, moderado o lento.

Combinando todas las posibilidades de movimiento rápido, moderado y lento tanto en la primera parte de la diástole como en la tercera parte tras la parada anómala en medio de la dilatación, se pueden dar nueve combinaciones. La anomalía consistiría en un retraso de la función pulsística (distribución del pneuma y mantenimiento del calor natural), porque la facultad (corazón) por unos instantes ve interrumpida su acción a causa de una lesión de los órganos (arterias) o por un exceso de humores.

No obstante, conviene tener en cuenta que, cuando las diástoles son pequeñas, no es posible percibir por el sentido del tacto pausa alguna, del mismo modo que los pulsos pequeños no se pueden dividir en dos partes, porque el tacto del médico no suele ser capaz de percibirlas. Si bien esto es lo que explica Galeno, dadas las circunstancias del estado tecnológico de la época, hoy se mide con precisión el pulso, con sus alteraciones y diferencias.

Por otro lado, en la anomalía de un solo pulso con interrupción de la diástole se debe tener en cuenta también las tres causas alteradoras que lo producen: uno, la abundancia desmedida de humores; dos, la presión que reciben los órganos (arterias) de las partes corporales que los rodean; y tres, la obstrucción (interna de la arteria) que puede impedir o entorpecer el movimiento circulatorio de la sangre.

De acuerdo con estas tres situaciones es posible observar que la facultad en el primer movimiento es presionada por una abundancia de humores, mientras que en el segundo movimiento que se produce tras la pausa el pulso se hace algo más lento que el primero; también se da el caso de que los dos movimientos anterior y posterior de la pausa, son rápidos, y hay un tercer tipo en el que el movimiento anterior a la pausa es rápido, mientras que el posterior es moderado (ni rápido ni lento). $Y$ añade otras variantes de pulsos en las que el primer movimiento anterior a la pausa es lento, y aquellas otras en las que ese primer movimiento es moderado; todo lo cual se resume en el siguiente cuadro de nueve combinaciones:

\begin{tabular}{|l|c|l|}
\hline \multicolumn{3}{|c|}{ DIÁSTOLE ANÓMALA } \\
\hline Movimiento 1 & Parada diastólica & Movimiento 2 \\
\hline 1: Rápido & - & lento \\
\hline 2: Rápido & - & rápido \\
\hline 3: Rápido & - & moderado \\
\hline 4: Lento & - & lento \\
\hline 5: Lento & - & rápido \\
\hline 6: Lento & - & moderado \\
\hline 7: Moderado & - & lento \\
\hline 8: Moderado & - & rápido \\
\hline 9: Moderado & - & moderado \\
\hline
\end{tabular}


Concluye Galeno que es peor el pulso que tiene lentas las dos partes de la diástole, y que es mejor el que las tiene rápidas, y es moderado el que las tiene en un término mediano. Ahora bien, cuanto mayor sea el tiempo de la pausa, mayor será la afección que padezca el cuerpo. Considera que lo mejor es la medida mediana, pero si solo hubiera las medidas extremas, la vehemencia sería mejor que la languidez, el tamaño mayor sería mejor que el menor, etc.

\section{PULSOS VIBRANTE Y DÍCROTO. CAUSA: DUREZA DE LA ARTERIA}

En el séptimo capítulo explica dos géneros de pulso que surgen también por una única anomalía. Galeno los denomina pulso vibrante y pulso dícroto. Ambos pertenecen a un tipo de pulsos que se caracteriza por sus vibraciones (pulso vibrante). Explica que el pulso vibrante propiamente dicho se caracteriza por disponer de una facultad vigorosa (corazón fuerte), un órgano duro (arteria) y una función (distribución del pneuma y del calor natural) que urge a la elaboración de los pulsos; por ello este tipo de pulsos exige que se den al mismo tiempo dos circunstancias: primera, que haya una diástole suficientemente enérgica que sea capaz de vencer la dureza de la arteria, y segunda, que esa energía en la diástole responda al vigor o fuerza que se transmite desde el corazón.

Si faltara una de estas dos circunstancias, se daría el caso de que el pulso no podría ser vibrante ni temblar: cuando solo la arteria está endurecida, pero la facultad no es vigorosa, no hay vibración pulsátil, sino dureza arterial al tacto; si la arteria no es dura, y la facultad es vigorosa, habrá un pulso grande, pero no habrá vibración ni dureza.

La causa continua de ese pulso vibrante es cuando la función no se completa. Se descarta, en consecuencia, por las propias características del pulso, que sea la debilidad de la facultad la causante del pulso (vibrante y dícroto), dado que, para que este pulso se dé, tiene que haber una facultad fuerte y vigorosa; podría suceder que los órganos (arterias) fueran insensibles a causa de las obstrucciones, opresiones o endurecimientos (esclerosis de órganos inflamados, escirróticos, endurecidos, resecos, enfriados o tensos). Con estas características el pulso que aparece es el vibrante.

Por otro lado, para que haya un pulso dícroto es necesario que la facultad sea vigorosa, que las arterias sean duras y, además, en lo cual se diferencia del pulso vibrante, que la función apremie la distribución del pneuma y la conservación del calor natural. El pulso dícroto se nota al tacto porque da la sensación de un pulso más duro y más frío, el cual a lo largo de la diástole toca el tacto, luego se retira y vuelve a tocar el tacto por segunda vez: es dícroto, porque golpea dos veces, mientras que en la parte central de la diástole se pierde su percepción. Tal pulso es dícroto, pero no vibrante. El pulso dícroto se produce por una gran descomposición (discrasia) y por algún endurecimiento (gordura, abundancia de humores o de carnes).

La diferencia esencial entre estos dos pulsos, vibrante y dícroto, es que en el pulso vibrante el golpe de la diástole en el tacto se repite varias veces, esto es, se observa que en una única dilatación la arteria, endurecida, toca el tacto varias veces. 
En el dícroto el movimiento de dilatación se siente claramente, pero se interrumpe una sola vez y la arteria, al reanudar su movimiento de dilatación, vuelve a golpear el tacto por segunda vez en el mismo movimiento de dilatación. En el pulso dícroto, por tanto, este rebote es único, lo que significa que la arteria golpea dos veces al tacto en un único latido.

\section{PULSO CAPRIZANTE. CAUSA: FACULTAD OBSTRUIDA U OPRIMIDA}

Como en los pulsos vibrantes y dícrotos, en el pulso caprizante la arteria golpea dos veces o más el tacto, pero la causa de ese movimiento doble en la diástole es distinta. En el pulso caprizante no tienen que ser los órganos (arterias) duros ni haberse producido en ellos una descomposición (discrasia), sino que lo que produce una interrupción momentánea de la diástole es la facultad (corazón), que mantiene correctamente la tensión natural, pero se ha alterado a causa de una abundancia de humores, de alguna obstrucción o de una opresión sobre los órganos (arterias); tras la primera interrupción o pausa dentro de un mismo movimiento diastólico, esa diástole prosigue su movimiento de dilatación y aparece al tacto como más vigorosa que en su primera parte; puede haber un segundo, tercero o más golpes en una sola diástole, con la particularidad de que esos sucesivos golpes son más fuertes que los anteriores, como Galeno reconoce; pero sea una o varias interrupciones las que tenga una diástole, estos pulsos se siguen denominando "caprizantes", y se diferencian de los vibrantes y dícrotos por el hecho de que en los caprizantes la facultad ve dificultada su acción por la abundancia de humores, obstrucciones y opresiones que dificultan la acción de las arterias.

\section{PULSOS ONDULANTE Y VERMICULANTE. CAUSA: FACULTAD DÉBIL Y ARTERIAS BLANDAS}

Otros dos pulsos anómalos se describen a continuación. Tienen en común el que la arteria es sentida por el tacto como si se curvara hacia arriba al moverse en una sola diástole; se diferencian en la cantidad: mientras el ondulante se encorva una sola vez en cada latido de forma semejante a la curva de una ola, el vermiculante se encorva varias veces imitando las ondulaciones de la piel de un gusano.

Dicha anomalía puede observarse en diferentes partes de la arteria. Galeno considera que estas dos anomalías son debidas a la debilidad de la facultad (corazón) por la abundancia de humores y por la blandura excesiva de las arterias. Esta situación provoca que la tensión no sea la misma en todo el recorrido arterial, lo que produce que en unas partes la tensión sea mayor que en otras.

Este caso significa que habrá partes de la arteria que no se podrán dilatar o moverse con la misma fuerza. Algunos médicos antiguos anteriores a Galeno interpretaban que estos pulsos primero se dilataban en las arterias más próximas al corazón y después se dilataban en las arterias más alejadas del corazón. Galeno rechaza 
esta creencia procedente de Erasístrato, porque ello equivaldría a decir que por el interior de las arterias correría libremente el pneuma o el fluido (arterial).

Galeno sostiene que el pulso normal en una persona sana late al mismo tiempo en todo el cuerpo. Si a veces parece que el flujo sanguíneo se detiene, luego prosigue, y de nuevo en otra parte de la arteria se detiene, y así sucesivamente a lo largo de toda la arteria, ello es consecuencia de una anomalía que da lugar a los llamados pulsos ondulante y vermiculante. En estos dos pulsos anómalos se da una alteración de la tensión, consecuencia de una facultad débil, porque el flujo sanguíneo es obstaculizado por la abundancia de humores y la blandura de las arterias; tras la interrupción del flujo sanguíneo y la blandura de las arterias, la tensión y la presión acumulada produce la reanudación del flujo sanguíneo. Mientras el pulso ondulante aparece por la debilidad de la facultad, el pulso vermiculante aparece por la abundancia de humores.

También se pueden observar estos dos pulsos cuando las arterias tienen exceso de humedad, lo que provoca que algunas arterias se doblen y se quiebren. Ello produce un pulso ondulante si la facultad está fuerte y levanta partes grandes de la arteria; pero si la facultad está débil el pulso será vermiculante y la tensión presentará pequeños intervalos.

Este pulso anómalo se puede observar en los hidrópicos, cuando aún tienen tensión y su pulso ancho y ondulante se hace anómalo y multiforme.

\section{EL PULSO MIURO EN UNA ANOMALÍA SIMPLE. CAUSA: FACULTAD DÉBIL, ARTERIA SECA}

Se produce siempre que la tensión se extiende desde el corazón hacia las arterias, pero dicha tensión se debilita en las partes más alejadas; ello significa que los movimientos en las arterias cercanas al corazón serán vehementes, mientras que los de las partes alejadas serán más lánguidos. Se niega la posibilidad de que pueda haber movimientos más pequeños cerca del corazón que lejos, porque es imposible también que la tensión inicial se debilite más en las partes arteriales cercanas al corazón que en las partes alejadas. En efecto, este caso guarda relación con la anomalía de la discrasia (o descomposición), de tal manera que la facultad sea mejor utilizada en beneficio de las partes bien constituidas y peor utilizada en las mal constituidas. De ahí que se considere mejor que el órgano (arteria) disponga de una humedad y temperatura moderadas para tener una tensión igual y la misma, mientras que el que esté exageradamente seco, húmedo o frío, hará que se mueva menos y más lentamente.

\section{VARIAS DISTORSIONES DE LOS PULSOS}

En los capítulos décimo y undécimo Galeno menciona de nuevo los pulsos miuros destacando que el movimiento pulsístico es mayor en relación con las partes que le preceden y le siguen, lo que denomina pulso miuro; esto no sucede cuando la 
facultad está muy fuerte, porque necesita estar inmóvil y no soporta la presión de lo que la rodea. Menciona otros dos pulsos que aún no define: innuente y circumnuente.

Se puede explicar la posición anómala de la arteria por una distorsión, tal como les sucede también a la matriz, al raquis y a los músculos cuando se tensan. La tensión puede ser debida a inflamaciones, escirros, abscesos y forúnculos, de la misma manera que la dureza seca, el enfriamiento desmedido, la congelación y la tensión espasmódica también pueden distorsionar las arterias.

\section{EL PULSO ESPASMÓDICO}

Dedica el capítulo décimo segundo al llamado pulso espasmódico. Se caracteriza porque la arteria se tensa hacia sus extremos y poco después sobreviene un espasmo al paciente. Galeno plantea que en este caso no se debe mezclar la tensión de la arteria, originada por el sistema circulatorio y percibida en las arterias, con el espasmo, originado en el sistema nervioso y cuya actividad se percibe por la "sensación" y por el movimiento voluntario. Fue Hipócrates quien afirmó que la naturaleza (en el organismo) es común, porque tiene una única corriente sanguínea, una única respiración y todas las partes sienten al mismo tiempo. Si esto no fuera así, seríamos no uno, sino varios animales. En efecto, mientras el corazón extiende las grandes arterias y se comunica con el cerebro, este extiende los nervios como vínculo de comunicación con el corazón. Del mismo modo las fibras nerviosas marchan hacia una comunicación con las arterias por partes, de tal forma que se intercambian acciones (pulsos) $\mathrm{y}$ afecciones (sensaciones nerviosas).

\section{EL TAMAÑO DE LOS PULSOS}

El capítulo décimo tercero está dedicado a hablar de los pulsos anómalos que destacan por su tamaño: altos y bajos, anchos y estrechos, largos y cortos.

En efecto, estos tipos de pulso se pueden observar en un único latido o en varios latidos sucesivos. Para ello hace falta mucha práctica en observar y distinguir un pulso de otro, con objeto de no confundirlos, sabiendo que lo natural es el pulso mediano en longitud, anchura y altura (o profundidad). Con sutileza, Galeno añade que al ser cilíndrica la arteria, en condiciones de normalidad y salud, las tres dimensiones citadas (longitud, anchura y altura) habrían de ser iguales teóricamente en un hombre sano, pero debe tenerse en cuenta también la situación de los cuerpos o partes que rodean la arteria, por lo que la diferencia en ser alto, ancho y estrecho no radica solo en la dimensión de la arteria en cada dilatación, sino que ha de tenerse en cuenta si esa arteria sobre la que se toma el pulso (generalmente en la muñeca) está sana o padece alguna afección. El pulso será anómalo desde el momento en el que haya una discrasia (o descomposición) del estado natural o un desvío en la posición. Indica que algunos pulsos en las discrasias de los órganos y en los desvíos están relacionados con determinadas afecciones y enumera algunas: por ejemplo, los pulsos altos son propios de pacientes con afecciones anímicas antes de entrar en crisis; los anchos se produ- 
cen cuando hay secreciones críticas, etc. Así pues, pueden ser cuatro las causas de esos pulsos: discrasia, desvíos de la posición arterial, afecciones psíquicas (pulsos altos, porque es su altura o profundidad la medida que mejor se percibe) y secreciones críticas (pulsos anchos, porque es la medida que mejor se percibe). Si dominara el pulso en dos dimensiones (altura y anchura) los denominará "grandes", porque entiende que un pulso es grande, no cuando ha aumentado en las tres dimensiones, sino cuando ha aumentado en dos de ellas.

Por tanto serán pulsos naturales (y normales) los que conserven una proporción natural, pero si esta no se conserva, los pulsos serán anómalos. Así se conservará el pulso natural cuando no sufra ningún gran cambio en cuanto a delgadez u obesidad en su constitución, ni haya sequedad excesiva, ni humedad abundante ni necesidad de una diástole extrema.

Si se diera alguno de estos cinco casos, se destruiría el pulso natural; aparecería el pulso alto y su aumento sería tal que nunca se estrecharía ni conservaría la proporción natural en las tres dimensiones. Por esta razón, el pulso alto es propio de los irritados.

\section{PULSO RARO}

Añade Galeno que muchos médicos perciben un pulso "raro", en el sentido común de "extraño", "desconocido", pero no lo describen ni lo denominan de ninguna forma, por lo que esos médicos demuestran que no saben distinguir las variantes de esos pulsos no habituales, a los cuales están acostumbrados a llamar "pulsos raros", sin tener en cuenta que la denominación "pulso raro" es una variedad específica de un pulso muy concreto, el opuesto al denominado pulso frecuente; con esa denominación inapropiada demuestran que no saben describir esas variedades de pulsos. Entre esas variantes está el pulso grande, pero no alto, propio de gente delgada y no fuerte.

\section{PULSO ANCHO}

Los pulsos anchos se producen cuando la facultad no está muy vigorosa y las zonas que rodean las arterias están extenuadas; también cuando la presión que ejercen algunas partes corporales sobre las arterias, el pulso parece más ancho que el moderado. Ese pulso se observa cuando la piel se ha vuelto rugosa, como en los ancianos y en los delgados.

\section{PULSO DE LOS HIDRÓPICOS}

Otro caso diferente es el que presentan los hidrópicos, quienes padecen un exceso de humedad. El resto de la explicación es la aplicación lógica de los excesos y deficiencias de la humedad. 


\section{PULSO LARGO}

Otro pulso es el denominado pulso largo, que se puede percibir sobre los cuatro dedos puestos sobre la muñeca, pero su movimiento es estrecho y oscuro como si fuera el énfasis de una cuerda delgada. La arteria vibraría por el golpe y el pulso se sentiría duro, además de estrecho y bajo, porque la facultad está más debilitada y el órgano aparece inflexible por su dureza.

Cuando el cuerpo humano es delgado, la piel que lo envuelve es más seca; cuando la piel es laxa y espesa, el movimiento se oculta mucho; lo contrario sucede cuando la piel se conserva delgada y tensa. Y al cuerpo delgado debe acompañar la sequedad de la piel, la dureza de los órganos y la debilidad no extrema de la facultad. Si fuera extrema, el hombre no tendría un pulso largo, ni siquiera tendría pulso. En efecto, cuando la facultad está debilitada completamente, no levanta la arteria hasta la altura que pueda ser percibida, esté duro o blando el conducto.

El pulso largo se denomina así por cierta excelencia, pero su nombre específico es "pulso delgado y grácil".

En cambio, el pulso verdaderamente largo es el delgado y notablemente grande, porque las otras dos dimensiones son comparativamente insignificantes.

\section{PULSO CORTO}

El pulso corto es el que tiene la arteria cubierta solo por la piel en una parte pequeña; ambas, piel y arteria, se hunden conjuntamente en profundidad. De manera que la parte que está bajo la piel muestra claramente todo el movimiento y las partes que están en lo profundo quedan completamente ocultas. Este pulso aparece en muchos cuerpos de forma natural y en unos pocos [aparece] por razones nocivas. También el pulso corto recibe los nombres de "espeso" y "grueso".

\section{PULSOS ORDENADOS Y DESORDENADOS}

En el capítulo décimo cuarto Galeno habla de los pulsos ordenados y desordenados, a los que dedicó una detallada atención en el libro titulado La diferencia de los pulsos ${ }^{3}$. Tanto unos como otros son pulsos anómalos; la diferencia está en el hecho de que el pulso ordenado es aquel que tiene una o varias anomalías que se repiten periódicamente de la misma manera; el pulso desordenado es el que tiene una

${ }^{3}$ Véase Pino Campos (2010), pp. 71-74, 208, 237: pulso ordenado, pulso desordenado:

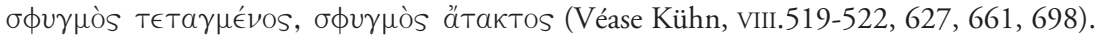


o varias anomalías que aparecen en distintas posiciones sin ninguna regularidad. La causa de esta doble diferencia es que la anomalía es firme en los pulsos ordenados, mientras que no lo es en los desordenados.

\section{EL RITMO DEL PULSO}

El capítulo décimo quinto está dedicado a explicar las causas que alteran el ritmo pulsístico. En otro lugar había explicado Galeno que a través de las arterias se transportaba y distribuía por todo el cuerpo una sustancia "aérea", es decir, un gas, que entraba en el cuerpo a través del aire que inspirábamos, y los movimientos de diástole y de sístole servían para atraer esas sustancias hacia el interior de las arterias, transportarlas y distribuirlas por todo el cuerpo $\mathrm{y}$, a su vez, recoger los residuos y sustancias fuliginosas y expulsarlas al exterior. Señala que cabría hacer una analogía entre los humores que el cuerpo produce y luego no utiliza, con los alimentos que una vez digeridos, debe expulsar los residuos por intestinos y vejiga; esta expulsión es comprobada por nuestros propios sentidos; en cambio, que las arterias transportan sustancias no se ve, pero sí se percibe por la razón.

\section{CONCLUSIÓN}

Hasta aquí Galeno ha explicado cada una de las causas primeras que alteran el pulso. Aún deberá explicar otras causas de alteración de los pulsos que son denominadas causas naturales, causas paranaturales y causas no-naturales, a las que dedica los libros tercero y cuarto del tratado.

En nuestros días contamos con sofisticados aparatos electrónicos que miden el pulso, número de latidos por minuto, ritmo, tamaño, frecuencia, tensión, etc., y que se registran digitalizando los resultados en gráficos de mayor o menor complejidad; este sistema ha sustituido totalmente a la antigua práctica de los médicos de tomar con las yemas de sus dedos el pulso de un paciente al palpar su arteria por la parte inferior de la muñeca, donde la arteria solo tiene por debajo la piel y sobre la arteria está solo el hueso.

Lo importante de este estudio es explicar, comprobar y admirar el esfuerzo de la medicina antigua para reconocer la enfermedad de un paciente a través del pulso entre otros síntomas, y elaborar una técnica tan sofisticada en el conocimiento del pulso como la que Galeno ha transmitido en sus siete tratados esfigmológicos conservados.

En un estudio anterior (el segundo citado sobre las causas de los pulsos) nos habíamos ocupado de presentar las que Galeno llamaba "causas generadoras de los pulsos", en un tercer estudio nos hemos ocupado de describir y comentar las llamadas causas alteradoras de los pulsos por varias anomalías simultáneas, mientras que en este nos hemos ocupado de sintetizar y comentar las denominadas causas alteradoras del pulso por una anomalía única. Están en prensa otros dos estudios sobre las causas no naturales y sobre las causas preternaturales de los pulsos. Estudios de las causas que se completan con los dedicados por el médico de Pérgamo a las diferencias, pronóstico y diagnóstico de los pulsos. 


\section{BIBLIOGRAFÍA (SELECCIÓN)}

BRAIN, P. (1986): Galen on bloodletting. A study of the origins, development and validity of his opinions, with a translation of the three works, Cambridge.

DAREMBerg, Charles (1879 - 1963'): "Recherches sur la sphygmologie antique", en Ch. DAREMBerG y Ch. E. Ruelle, Oeuvres de Rufus d’Ephèse, París, 1879, Amsterdam, 1963, pp. 219-232.

DeichgräBer, Karl (1956 - 1984): "Galen als Erforscher des menschlichen Pulses. Ein Beitrag zur Selbstdarstellung des Wissenschaftlers (De dignotione pulsuum, I.1)”, SB d. Deutschen Akademie d. Wiss. Kl.f. Sprachen, Lit. u. Kunst 3: 1-41 [Berlín 1957] [= H. GÄrTnER, E. Heitsch, U. SCHINDEL (1984): Ausgewählte Kleine Schriften, Hildesheim, Munich, Zurich, pp. 288-326].

FLEMING, D. (1955): "Galen on the motions of the blood in the heart and lungs", Isis 46: 14-21.

Furley, David J. - J. S. WILKIE (eds.) (1984): Galen: On respiration and the arteries. An edition with English translation and commentary of De usu respirationis; An in arteriis natura sanguis contineatur; De usu pulsuum, and De causis respirationis, Princeton U.P. [pp 194-227] [Edición bilingüe greco-inglesa].

Garofalo, Ivan (1998): "Le commentaire ravennate au De pulsibus ad tirones de Galien: le commentaire et ses sources”, en C. DEROUX (ed.): Maladie et maladies dans les textes latins antiques et médiévaux, Bruxelles (Collection Latomus, vol. 242), pp. 382-392.

Garofalo, I., - M. Vegetti (1978): Opere scelte di Galeno. Turín.

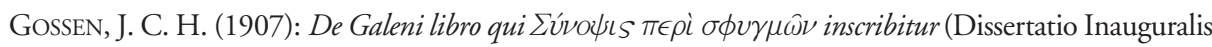
Philologica), Berlín.

Gotfredsen, E. (1942): “Oldtidens Laere om Hjerte, Kar og Puls", Acta Hist. Scient. Nat. Med. 1, Copenhague, pp. 317-42.

HARRIS, C. R. S. (1973): The heart and vascular system in ancient Greek Medicine from Alcmeon to Galen, Oxford [267-431; 440-455].

Horine, E. F. (1941): "An Epitome of Ancient Pulse Lore”, Bulletin of the History of Medicine X.1: 209-249.

López Férez, Juan Antonio (1991): Galeno: obra, pensamiento e influencia, UNED, Madrid.

MAY, Margaret Tallmadge (1968): Galen. On the Usefulness of the Parts of the Body, Cornel University Press, Ithaca, Nueva York, dos vols.

Mitaritonna, O. (1968): "Il pensiero di Galeno sul movimento del cuore", Collana di Pagine di Storia della Medicina, Miscell. 19: 133-143.

NiebyL, P. H. (1971): “The non-naturals causes”. Bull. Hist. Med. 45: 486-492.

Palmieri, Nicoletta (1999): Agnellus de Ravenne, Commentaire sur le De pulsibus ad tirones de Galien. Intr. texte latin. (Adiuuante I. GAROFALO), traduction, commentaire par... Thèse d'habilitation, Grenoble.

Pino CAmpos, Luis Miguel (2004): "Léxico esfígmico antiguo y su pervivencia en nuestros días", en J. A. LÓPEZ FÉREZ (ed.), La lengua cientifica griega: orígenes, desarrollo e influencias en las lenguas modernas europeas. III, Ediciones Clásicas, Madrid, pp. 209-238.

(2005a): Galeno: Sinopsis de Galeno de su propia obra sobre pulsos, Ediciones Clásicas, Colección Obras de Galeno: Gal. 5, Madrid.

- (2005b): "La doctrina galénica del pulso: síntesis del libro Sobre la utilidad de los pulsos", Actas del XI Congreso Español de Estudios Clásicos, Madrid, v. II, pp. 477-486. 
- (2006): "Observaciones al tratado de Galeno Acerca de la diferencia de los pulsos, Fortunatae 17: 99-115.

(2010): Galeno. Sobre la diferencia de los pulsos, Ediciones Clásicas, Colección Obras de Galeno: Gal. 7, Madrid.

(2015a): Galeno. Los pulsos para principiantes. La utilidad de los pulsos, Ediciones Clásicas, Colección Obras de Galeno: Gal. 10, Madrid.

_ (2015b): “Apuntes en torno a la esfigmología galénica: la Sinopsis”, en J. A. LópEz FÉrEZ, Galeno. Lengua, composición literaria, léxico, estilo, UNED, Madrid, pp. 315-342.

— (2016): "Doctrina de Galeno sobre las causas de los pulsos. II: Introducción, casuística y comentarios", Fortunatae 27: 119-144.

- (2017a): "Consideraciones en torno al tratado galénico De causis pulsuum", en Actas del XIV CEEC [Barcelona, 6-10 de julio de 2015], SEEC, Madrid, vol. I, pp. 621-629.

_ (2017b [en prensa]): "Doctrina de Galeno sobre las causas de los pulsos. III: casos y comentarios», en Homenaje a Luis Miguel Macía Aparicio, Univ. Aut. Madrid, Madrid.

Ruiz Moreno, Aníbal y Antonio Tovar (1948): Obras de Galeno, Publicaciones del Instituto de Historia de la Medicina (Facultad de Ciencias Médicas), vol. XII, t. I, Buenos Aires [Contiene los textos titulados Compendio del pulso para los estudiantes y De las diferencias de pulsos].

SÁnCHEZ Granjel, Luis (1961): "La circulación de la sangre según Galeno”, Boletín de la Sociedad Española de Historia de la Medicina, 1.2: 4 ss.

SCHADEWALDT, Otto (1866): Sphygmologiae historia inde ab antiquissimis temporibus usque ad aetatem Paracelsi, Tesis, Berlín. 